\title{
Weichselzeitlicher Löß als Rohstoff für die landwirtschaftliche Rekultivierung im Rheinischen Braunkohlentagebau
}

\author{
Rainer Schmidt \& Armin Skowronek*)
}

Weichselian loess, agriculture reclamation, soil physics, soil chemistry, Rhine Browncoal Area

\begin{abstract}
Kurzfassung: Im Rheinischen Braunkohlentagebau werden seit dem 19. Jahrhundert große Flächen der landwirtschaftlichen Nutzung entzogen und nach der Auskohlung einer Wiedernutzbarmachung zugeführt. Aufgrund der reichen Lößvorkommen in der Niederrheinischen Bucht besteht die Möglichkeit, agrarische Nutzflächen mit guten bodenkundlichen Eigenschaften herzustellen. Die Untersuchung eines im Tagebau Garzweiler I anstehenden Lößprofils soll die bodenphysikalisch/-chemischen Verhältnisse aufzeigen und zu einer differenzierten Beurteilung der Rekultivierungseignung der Lösse führen. Anhand der Ergebnisse lassen sich auch Rekultivierungs- und Bearbeitungseffekte in Neulandböden (Kultosolen) besser einschätzen.
\end{abstract}

\section{[Weichselian loess as natural resource for agriculture reclamation in the Rhine Browncoal Area]}

\begin{abstract}
Since the 19th century in the Rhine Browncoal Area large lands are eliminated for agricultural treatment and reclaimed after the coal-mining. Because of the abundant loess in the Lower Rhine Basin it is possible to reclaim agricultural lands with good soil qualities. Soil physical/chemical properties are shown by the investigation of a loess profile in the browncoal mine Garzweiler I. It provides a valuation for the aptitude of the loess. With these results it is even possible to evaluate the effects of the reclamation and lands treatment.
\end{abstract}

\section{Einleitung}

Unter den mineralischen Rohstoffen spielt der pleistozäne Löß wertemäßig eine untergeordnete Rolle, da er im wesentlichen nur in der Ziegelherstellung Verwendung findet (EGGERT et al. 1986: 356). Große Bedeutung erlangt er jedoch dort, wo man andere oberflächennahe Lagerstätten im Tagebau ausbeutet und dazu vorher wertvolle Ackerböden aus Löß ab-

\footnotetext{
*) Anschrift der Verfasser: Dipl.-Ing. Agr. R. Schmidt und Prof. Dr. A. SKOwronek, Institut für Bodenkunde der Universität, Abt. Experimentelle Bodenkunde, Nußallee 13, 53115 Bonn

*) Erweiterte Fassung eines Vortrags des Erstautors am 16. September 1992 in Kiel anläßlich der 26. Jahrestagung der Deutschen Quartärvereinigung.
}

getragen werden müssen: hier ist er das am besten geeignete Rekultivierungsmaterial für die Wiedernutzbarmachung der entstandenen Bergbauflächen. Die im Bereich des Rheinischen Braunkohlentagebaus vorkommenden Lösse werden deshalb überwiegend zur Herstellung agrarisch genutzten Neulandes verfügbar gemacht.

Der großtechnische Abbau im Rheinischen Revier hat eine erhebliche Ausdehnung erreicht und schon über 23000 ha Land beansprucht (s. Abb. 1). Die bis zum Jahr 2020 geplante und genehmigte Förderung wird weitere 10850 ha Fläche benötigen. Flächenbilanzen lassen erkennen, daß hauptsächlich wieder die Landwirtschaft betroffen sein wird (Tab. 1). Die landwirtschaftliche Rekultivierung und das Rekultivierungsmaterial müssen daher auch in Zukunft beachtet werden.

Nach den "Richtlinien des Landesoberbergamts Nordrhein-Westfalen für das Aufbringen von kulturfähigem Bodenmaterial bei landwirtschaftlicher Rekultivierung für die im Tagebau betriebenen Braunkohlenwerke" sollen nur die zuoberst anstehenden Lößlehme, also die holozänen, nicht-hydromorphen Oberflächenböden, und der darunter folgende kalkhaltige Weichsellöß verwendet werden (LANDESOBERBERGAMT NW 1973). Die Gewinnung erfolgt mit Schaufelradbaggern in einer Mächtigkeit von 4 bis $6 \mathrm{~m}$. Dann wird das Bodenmaterial auf Förderbändern zum Bestimmungsort transportiert und dort verkippt. Unmittelbar nach der Planierung beginnt die landwirtschaftliche Erstbewirtschaftung. Die so hergestellten Kultosole (vgl. AG BODENKUNDE 1982: 262-263) werden durch den Bergbautreibenden für etwa 7 Jahre zwischenbewirtschaftet, bevor sie der Bodenschätzung unterzogen werden.

Da Entwicklung und Bewertung rekultivierter Lößböden in mehrfacher Hinsicht problematisch werden können (SCHRÖDER \& STEPHAN \& SCHUlTEKARRING 1985), auf der anderen Seite aber bisher nur sehr wenige Qualitätsuntersuchungen von anstehenden Lössen vorliegen, soll im folgenden der Frage nachgegangen werden, wieweit primäre Lößmerkmale die Eigenschaften und die Entwicklung 


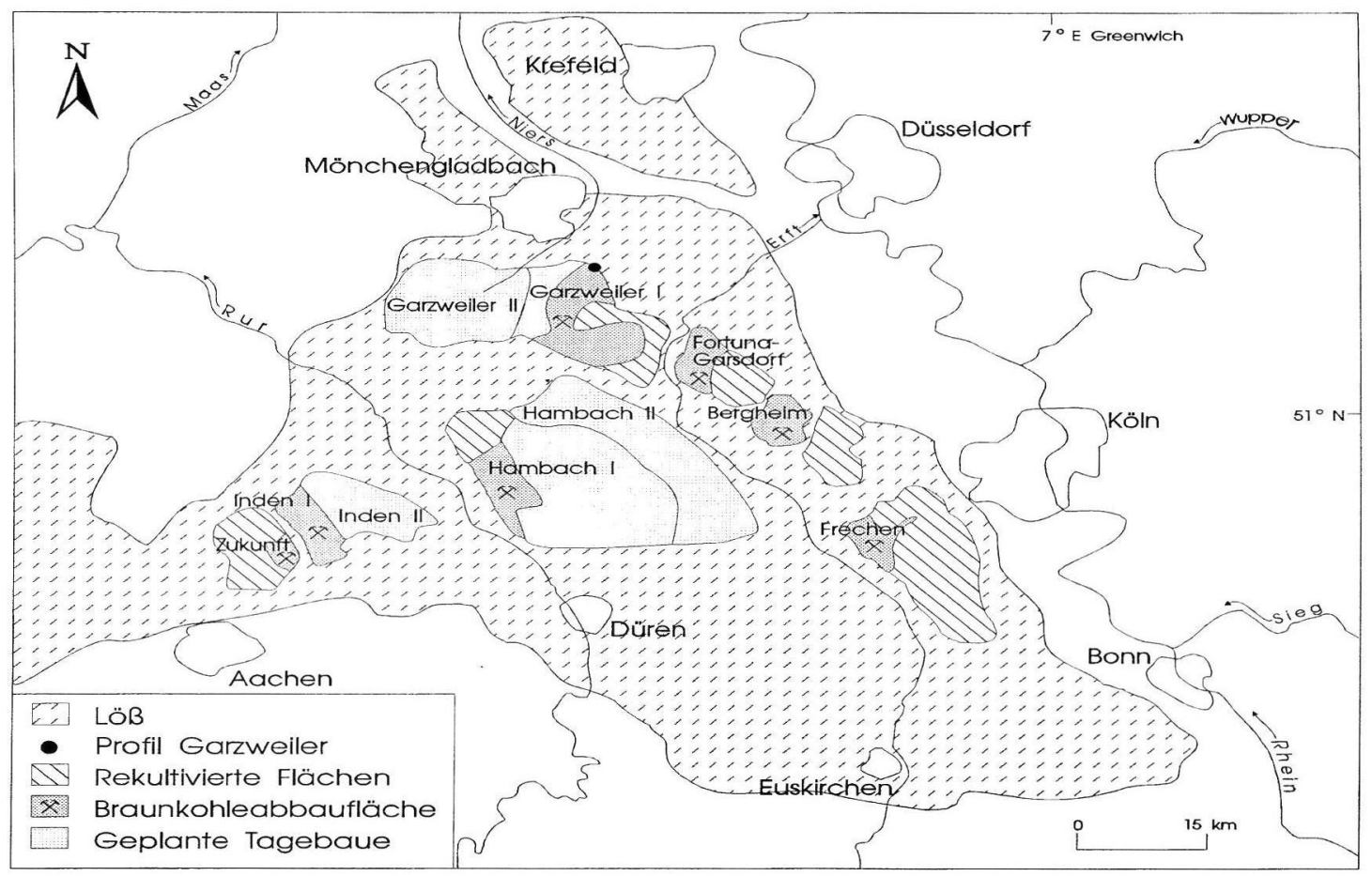

Abb. 1: Ausdehnung des Rheinischen Braunkohlentagebaus und linksrheinische Lößverbreitung.

Tab. 1: Flächenbilanz im Rheinischen Braunkohlenrevier für 1990 und 2020 in ha (aus STARKE 1990:9)

\begin{tabular}{|l|r|r|r|r|r|}
\hline & $\begin{array}{c}\text { Landwirt- } \\
\text { schaft }\end{array}$ & $\begin{array}{r}\text { Forstwirt- } \\
\text { schaft }\end{array}$ & $\begin{array}{r}\text { Wasser- } \\
\text { flächen }\end{array}$ & $\begin{array}{r}\text { sonstige } \\
\text { Flächen }\end{array}$ & Summe \\
\hline Stand: 1.1.1990 & & & & & \\
Landinanspruchnahme & 15320 & 6350 & 70 & 1460 & 23200 \\
Wiedernutzbarmachung & 6490 & 6270 & 600 & 1010 & $\begin{array}{r}14370 \\
8830\end{array}$ \\
Betriebsfläche & & & & & \\
\hline Stand: 1.1.2020 & 23000 & 8580 & 80 & 2390 & 34050 \\
& 15010 & 8990 & 740 & 1260 & 26000 \\
Landinanspruchnahme & & & & & \\
Wiedernutzbarmachung & & & & & \\
Betriebsfläche & & & & & \\
\hline
\end{tabular}

junger Kultosole zu beeinflussen vermögen. Dazu werden bodenphysikalische und bodenchemische Untersuchungsergebnisse vorgestellt, die an einem für die Rekultivierung vorgesehenen Löß gewonnen wurden. Über den Vergleich mit Gemischen aus Lößlehm und carbonathaltigem Löß, mit Kültosolen aus Löß und mit holozänen Lößböden erfolgt dann eine differenzierte Bewertung der Rekultivierungseignung des untersuchten Weichsellösses.

\section{Der Löß im Tagebau Garzweiler I}

Der Aufschluß von Garzweiler liegt im nördlichen
Teil einer zusammenhängenden Lößdecke, die zwischen Krefeld, Bonn und Aachen dreiecksförmig die südliche Niederrheinische Bucht erfüllt (s. Abb. 1 u. Klostermann 1992: Abb. 28). Am Nordoststoß erkennt man eine mehr oder weniger gleichbleibende Mächtigkeit des hangenden Lösses von 8 bis $10 \mathrm{~m}$. Die Sedimente liegen hier unmittelbar den groben Quarzschottern einer Hauptterrasse des Rheins auf. Verfärbungshorizonte unter der holozänen $\mathrm{Pa}$ rabraunerde zeigen an, daß die Lößakkumulation mehrfach von einer Bodenbildung unterbrochen wurde. An einigen Stellen wurde eine fossile, wahr- 
scheinlich die eemzeitliche Parabraunerde gefunden, welche im Saalelöß, aber auch im liegenden Rheinschotter entwickelt ist. Der Lößvorrat im Tagebau Garzweiler I wird auf $180 \mathrm{Mio.} \mathrm{m}^{3}$ veranschlagt (Hochт 1990: Tab. 3).

Die Beprobung des Lößpaketes erfolgte an einer Stelle, die wenig später zur Gewinnung von Rekultivierungsmaterial abgebaut wurde und die keinen fossilen Bt-Horizont aufwies, die also im Sinne des LANDESOBERBERGAMTS NW (1973) nur Lößlehm und kalkhaltigen Löß enthielt. Das Profil, dessen Oberfläche bei $94 \mathrm{~m}$ ü. NN lag und ackerbaulich genutzt wurde, war wie folgt aufgebaut.

\section{Tiefe Horizont Kurzbeschreibung}

$\mathrm{cm}$

0-33 Ap dunkelbrauner (10 YR 3/4), carbonatfreier, stark lehmiger Schluff; Krümelgefüge

- $60 \mathrm{Bt}$

mattbrauner (7.5 YR 5/4), carbonatfreier, stark lehmiger Schluff, deutliche Tonanreicherung; Polyedergefüge

hellbrauner (7.5 YR 4/6), carbonatfreier, stark lehmiger Schluff; sehr schwach ausgeprägtes Polyedergefüge

brauner (7.5 YR 4/6), carbonatfreier, stark lehmiger Schluff, mit Tonbändern; Subpolyedergefüge

gelblich brauner (10 YR 5/6), carbonatfreier, stark lehmiger Schluff; Subpolyedergefüge

$>860$ IIC hell gelblich brauner (10 YR 6/6), carbonatreicher, mittel lehmiger Schluff; Kohärentgefüge

$-700 \quad 2 . \mathrm{fGoC}$

hell gelblich brauner (10 YR 6/8), carbonatreicher, stark lehmiger Schluff, oben stark kryoturbat verwürgt; $z$. T. schwach plattiges Gefüge

\section{-745 GoCv}

$-780 \quad$ GroCv

$$
-860 \quad 3 . \mathrm{fBv}
$$

gelblich brauner (10 YR 5/6), carbonatreicher, stark lehmiger Schluff, stellenweise MnKonkretionen; Kohärentgefüge

gelblich brauner (10 YR 5/6), carbonathaltiger, stark lehmiger Schluff, Fe- und Mn-Konkretionen; Kohärentgefüge

hellbrauner (7.5 YR 5/8), stark lehmiger Schluff; durch Umlagerung beeinflußtes Kohärentgefüge

Schotter der Rheinhauptterrasse

Der unterste fossile Horizont (3.fBv) würde in der stratigraphischen Gliederung von PAAs (1968: Tab. 2) einer "braunerdeartigen Bodenbildung" der älteren Weichselzeit entsprechen, die darunter auch noch Humuszonen aufweisen kann. Die hangenden fossilen Bodenhorizonte sind danach genetisch als Tundragleye anzusprechen und gehören sowohl zum älteren als auch zum jüngeren Weichsellöß.

Daß an der Probenentnahmestelle in Garzweiler I nicht die maximal mögliche Anzahl fossiler Weichselböden erhalten ist, zeigen Sammelprofile aus dem Rheinland von BRunNaCkER \& HAHN (1978: Abb. 1) mit 3 Humuszonen an der Basis, gefolgt von 2 bis 3 Verbraunungszonen und 3 Naßböden. In der überregionalen stratigraphischen Korrelation von RICKEN (1983: Abb. 11) könnte der 3.fBv-Horizont identisch sein mit dem Niedervellmarer Bodenkomplex (Mittelweichsel) Nordhessens bzw. Südniedersachsens, der 2. fossile Gley mit dem Kirchberger Boden (ebenfalls Mittelweichsel) oder bereits mit einem der Jungweichsel-Naßböden, denen zumindest der 1 . fossile G-Horizont angehören dürfte. Mangels geeigneter Zeitmarken (Eltviller Tuff) kann das aber nicht sicher entschieden werden.

\section{Laboranalytische Kennzeichnung}

Da rekultivierte Böden in ihrer Leistungs- und Ertragsfähigkeit gewachsenen Böden entsprechen sol- 


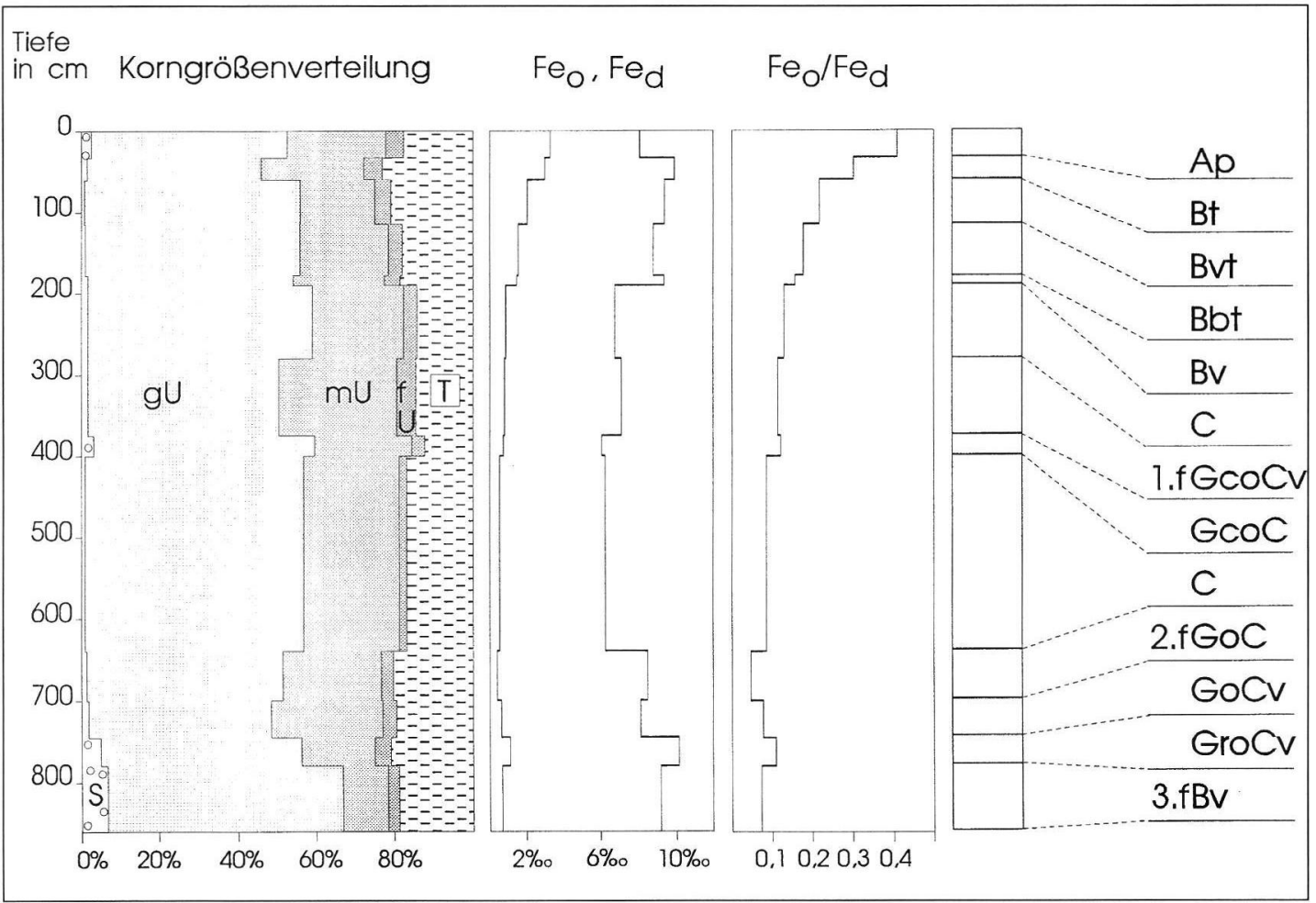

Abb. 2: Tiefenfunktionen der Korngrößen, des röntgenamorphen $\left(\mathrm{Fe}_{0}\right)$ und gesamten freien Eisens $\left(\mathrm{Fe}_{\mathrm{d}}\right)$ sowie Aktivitätsgrad $\left(\mathrm{Fe}_{0} / \mathrm{Fe}_{\mathrm{d}}\right)$ des Profils Garzweiler

len (LANDesoberbergamt NW 1973), wird das Rekultivierungsmaterial, hier Weichsellösse, mit den gleichen Methoden untersucht wie Naturböden. Im folgenden werden daher die Ergebnisse einiger bodenphysikalischer und -chemischer Standardanalysen des Lößprofils Garzweiler mitgeteilt.

\subsection{Bodenphysikalische Eigenschaften}

Der Aufnahme bodenphysikalischer Parameter kommt erhöhte Bedeutung zu, weil diese die bodenchemischen und -biologischen Eigenschaften beeinflussen können, und weil im Rheinischen Braunkohlenrevier außer Korngrößenanalysen u. W. bisher keine weiterführenden bodenphysikalischen Untersuchungen an Lößprofilen durchgeführt wurden (vgl. HeIDE 1954: 93-94 od. Носнт 1990: Tab.2).

\section{Körnung}

Die Korngrößenverteilung im Profil Garzweiler spiegelt sowohl die geologische Entwicklung der Lösse als auch deren pedogenetische Überprägung wider (Abb. 2). Periglaziale und kolluviale Umlagerungen werden durch wechselnde Sandgehalte (S), aber auch durch unterschiedliche Grobschluffanteile (gU) angezeigt. Die Bodenart bleibt aber immer ein lehmiger Schluff (Ul). Der Grobschluff dominiert absolut, seine mengenmäßige Verringerung geht zumindest in der holozänen Parabraunerde ("Lößlehm") und im 2. fossilen Boden (Tundragley) auf pedogene Tonbildung zurück. Dafür spricht auch der Verlauf des dithionitlöslichen Eisens $\left(\mathrm{Fe}_{\mathrm{d}}\right.$ ), während die oxalatlösliche Fraktion $\left(\mathrm{Fe}_{\mathrm{o}}\right)$ die pedochemische Tonbildung nicht mehr so stark nachzeichnet, vermutlich weil das röntgenamorphe $\mathrm{Fe}_{\mathrm{O}}$ postpedogen gealtert ist (vgl. dazu BRONGER 1974). Der sog. Aktivitätsgrad des Bodeneisens, ausgedrückt durch das Verhältnis von $\mathrm{Fe}_{\mathrm{O}} \mathrm{zu} \mathrm{Fe}_{\mathrm{d}}$, ist deshalb im holozänen Lessivé am höchsten.

Die von den Sedimentationsbedingungen, von sekundären Umlagerungen und von Bodenbildungen verursachten Inhomogenitäten der Textur hat auch HeIDE (1954: 39-55) an zwei Aufschlüssen und an vier Trockenbohrungen im benachbarten Tagebau Fortuna beschrieben. Trotzdem erweist sich die Korngrößenzusammensetzung wegen der hohen Schluffgehalte ( $>60 \%$ ) hier als sehr günstig für die Rekultivierung. Im Tagebau Inden werden von Hоснт (1990: Tab. 2) im Weichsellöß (Schicht 3 u. 4) und in den aufliegenden Kolluvien vergleichbare Bodenarten festgestellt. 
Tab. 2: Bodenphysikalische Parameter

\begin{tabular}{|c|c|c|c|c|c|c|c|c|}
\hline \multirow[t]{2}{*}{ Horizont } & Tiefe & $\begin{array}{l}\text { Lagerungs- } \\
\text { dichte }\end{array}$ & $\begin{array}{l}\text { Gesamtporen- } \\
\text { volumen }\end{array}$ & $\begin{array}{l}\text { Poren } \\
>50 \mu \mathrm{m}\end{array}$ & $\begin{array}{c}\text { Poren } \\
50-10 \mu \mathrm{m}\end{array}$ & $\begin{array}{c}\text { Poren } \\
<10-0,2 \mu \mathrm{m}\end{array}$ & $\begin{array}{l}\text { Poren } \\
<0,2 \mu \mathrm{m}\end{array}$ & \multirow{2}{*}{$\begin{array}{c}\begin{array}{c}\text { Ges. Wasserleit- } \\
\text { fähigkeit }\end{array} \\
\mathrm{cm} / \mathrm{d} \\
\end{array}$} \\
\hline & $\mathrm{cm}$ & $\mathrm{g} / \mathrm{cm}^{3}$ & \multicolumn{5}{|c|}{ Vol.-\% } & \\
\hline Ap & $0-33$ & 1,44 & 49,7 & 13,6 & 5,5 & 18,7 & 11,9 & 76,9 \\
\hline $\mathrm{Bt}$ & -60 & 1,55 & 44,2 & 6,8 & 4,3 & 18,4 & 14,7 & 55,3 \\
\hline Bvt & -115 & 1,50 & 46,7 & 6,0 & 5,6 & 22,5 & 12,6 & 32,8 \\
\hline Bbt & -178 & 1,41 & 51,4 & 8,0 & 9,3 & 23,3 & 10,8 & 77,8 \\
\hline $\mathrm{Bv}$ & -190 & 1,34 & 55,9 & 11,1 & 13,9 & 20,9 & 10,0 & 267,8 \\
\hline C & -280 & 1,41 & 52,7 & 7,9 & 13,0 & 22,2 & 9,6 & 22,6 \\
\hline 1.fGcoCv & -375 & 1,46 & 52,2 & 5,7 & 8,6 & 28,0 & 9,9 & 25,1 \\
\hline $\mathrm{fGcoC}$ & -400 & 1,40 & 54,3 & 7,1 & 14,4 & 25,0 & 7,8 & 24,2 \\
\hline C & -640 & 1,44 & 54,6 & 7,2 & 11,9 & 29,0 & 6,5 & 17,3 \\
\hline 2.fGoC & -700 & 1,51 & 53,8 & 3,9 & 6,6 & 34,2 & 9,1 & 14,7 \\
\hline $\mathrm{fGoCv}$ & -745 & 1,48 & 49,2 & 3,0 & 3,2 & 31,9 & 11,1 & 18,1 \\
\hline fGroCv & -780 & 1,56 & 45,6 & 4,1 & 4,4 & 25,3 & 11,8 & 19,9 \\
\hline 3. $f B v$ & -860 & 1,55 & 46,0 & 3,8 & 8,9 & 23,1 & 10,2 & 57,0 \\
\hline
\end{tabular}

L a gerungsdich te

Sie reicht von 1,34 bis $1,56 \mathrm{~g} / \mathrm{cm}^{3}$ (Tab. 2) und liegt damit in einem Bereich, der für Lösse mit einem Kornverteilungsmaximum bei $30 \mu \mathrm{m}$ Durchmesser typisch ist (vgl. Rohdenburg \& Meyer 1966: Tab. 8). Die Hohlraumveränderungen gegenüber der lockersten Schüttung $\left(1,34 \mathrm{~g} / \mathrm{cm}^{3}\right)$ gehen auf Sackungsund Einlagerungsverdichtung zurück, sicher werden aber auch Kompaktierungen infolge Kryoturbation zu berücksichtigen sein. Zumindest für den 1.fGcoCv- und den 2.fGoC-Horizont ist diese auch makroskopisch im Aufschluß nachzuweisen (s. o.). Ebenso erzeugte der Wechsel von Gefrieren und Auftauen über Preß- und Knetvorgänge Verdichtungen, welche die unterschicdlichen Raumgewichte unterhalb der holozänen Parabraunerde bedingen. In letzterer haben Tondurchschlämmung und Bodenbearbeitung zu einer Erhöhung der Lagerungsdichten beigetragen. Ähnliche Lagerungsdichten sind aus der Normandie bekannt, wo die Dichte trockener Lösse zwischen 1,38 und $1,69 \mathrm{~g} / \mathrm{cm}^{3}$, die nasser Lösse zwischen 1,60 und 2,04 g/ $\mathrm{cm}^{3}$ schwankt (LAUTRIDOU \& MASSON \& VOIMENT 1987: 18).

Unter Berücksichtigung des Tongehaltes kann die effektive Lagerungsdichte rechnerisch ermittelt werden aus: $\mathrm{Ld}_{\text {eff }}=\mathrm{Ld}+0,009 \mathrm{~T}(\mathrm{Ld}=$ Lagerungsdichte; $\mathrm{T}=$ Tongehalt) (AG BODENKUNDE 1982: 128). Für das Profil Garzweiler ergeben sich daraus Werte von 1,50 (Bv u. fGcoC) bis $1,75(\mathrm{Bt})$

Porung

Sie läßt sowohl in allen Wasserspannungsbereichen als auch im Gesamtporenvolumen z. T. erhebliche vertikale Unterschiede erkennen. Die sekundär entstandenen Grobporen (Durchmesser $>10 \mu \mathrm{m}, \mathrm{pF}<$ 2.5) besitzen im Bv-Horizont des Holozänbodens ein Maximum, mit 6.2\% im GoCv-Horizont (2.f) ein Minimum. Generell nimmt dieser für die Wasserleitfähigkeit wichtige Porenanteil mit zunehmendem Alter der Lösse ab. Die geringeren Gehalte im Btund Bvt-Horizont der Parabraunerde sind dagegen durch Einlagerung von Ton in die Grobporen zu erklären.

Eine gegenläufige Tendenz ist bei den Mittelporen (Durchmesser 10 - 0,2 um, pF 2,5-4,2) festzustellen, während die Gehaltsunterschiede der Feinporen (Durchmesser $<0,2 \mu \mathrm{m}, \mathrm{pF}>4,2$ ) offensichtlich wieder mit den jeweiligen Tonanteilen korrespondieren (vgl. Abb. 2). Der für die Wasserversorgung von Kulturpflanzen entscheidende mittlere Porenbereich scheint dagegen mehr vom pedogenetischen $\mathrm{Zu}-$ stand bzw. vom Kalkgehalt abzuhängen: das lockere Schüttungsgrundgefüge von Lössen erzeugt wegen der hohen (Grob-)Schluffmengen auch einen verhältnismäßig großen Mittelporenraum, der durch primäres und/oder sekundäres Carbonat sowie durch Ton stabilisiert wird. Nur mechanische Durchknetung bei höherer Feuchte führt zur Reduktion dieses Hohlraumanteils. Damit können Lösse stärker komprimiert werden als andere Lockersedimente (Rohdenburg \& MEyer 196: 98).

Gesättigte Wasserleitfähigkeit

Als Materialeigenschaft hängt die Wasserleitfähigkeit des Bodens sehr stark von Porenform und -kon- 
tinuität ab, so daß vor allem die Bodenstruktur bzw. die Ausprägung der Sekundärporen über die Höhe der Permeabilität entscheiden. Die Unterschiede der vertikalen gesättigten Wasserleitfähigkeit im Profil Garzweiler stehen in deutlicher Beziehung zu den jeweiligen Summenanteilen der schnell (Durchmesser $>50 \mu \mathrm{m}$ ) und der langsam (Durchmesser $50-10$ $\mu \mathrm{m})$ dränenden Grobporen, doch nicht gleichmäßig, weil auch deren Kontinuität verschieden ist. Das erkennt man u. a. an der sehr hohen, mehrfach gemessenen Permeabilität des holozänen $\mathrm{Pa}$ rabraunerde-Bv-Horizontes $(267,8 \mathrm{~cm} / \mathrm{d})$, welche um den Faktor 3,4 bzw. 18,2 größer als die zweithöchste $(77,8 \mathrm{~cm} / \mathrm{d})$ bzw. die niedrigste $(14,7 \mathrm{~cm} / \mathrm{d})$ Wasserleitfähigkeit ist. Auch eine Gegenüberstellung von Permeabilität und Porendurchmesser $>50$ $\mu \mathrm{m}$ belegt die vertikalen Porendiskontinuitäten.

Insgesamt gesehen scheinen die bodenphysikalischen Eigenschaften von Profil Garzweiler überwiegend pedogen oder postgenetisch erworben zu sein, da die Akkumulationen carbonathaltigen Lösses mengenmäßig so viel Grobschluffkomponenten enthielten, daß von einer weitgehend homogenen Primärdisposition der Sedimente ausgegangen werden kann. Die Erkenntnis, daß mächtige Lößdecken dennoch bodenphysikalisch differenziert sind, erweist sich auch in der Praxis als sehr nützlich (vgl. z. B. Hase \& Meyer 1969, Luft 1980 od. Lautridou \& Masson \& VoIment 1987).

\subsection{Bodenchemische Eigenschaften}

Die mengenmäßig von den Liefergebieten abhängigen und während der äolischen Ablagerung homogenisierten Lößkomponenten (TILLMANNS \& BRUNNACKER 1987: 53) alterieren in Bodenbildungsphasen, welche vor allem durch Carbonatmetabolik ausgezeichnet sind. Tieferreichende Silikatverwitterung ist jedoch an das Absinken der Kalkgrenze gebunden (ROHDENBURG \& MÉYER 1966: 72).

Im Profil Garzweiler lassen sich daher verschieden starke Intensitäten der Pedogenese am $\mathrm{CaCO}_{3}$-Gehalt ablesen (Tab. 3). Dabei muß aber auch die Möglichkeit einer Bildung von Sekundärcarbonat in Betracht gezogen werden, was auch schon bei der feldbodenkundlichen Untersuchung geschah. Demzufolge sind die pH-Werte $\left(\mathrm{CaCl}_{2}\right)$ in der entkalkten, aber ackerbaulich genutzten (!) Oberflächenparabraunerde niedriger als in den primär kalkhaltigen bzw. sekundär mit $\mathrm{CaCO}_{3}$ angereicherten Fossilböden. Die kleineren Gehalte im 2. fossilen Tundragley sprechen zusammen mit einer höheren effektiven Kationenaustauschkapazität für eine intensivere Mineralverwitterung und Verlehmung, was auch durch die verstärkte Tonbildung und Eisenfreisetzung unterstrichen wird (s. Abb. 2). Das läßt möglicherweise eine stratigraphische Zuordnung in das Mittelwürm
(Kirchberger Boden ?) zu, weil in diesem Abschnitt die pedogene Überprägung mit der Lößakkumulation Schritt halten konnte (vgl. ROHDEnBurg \& Meyer 1966: 31).

Die $\mathrm{KAK}_{\text {eff }}$ ist nicht besonders hoch. Bei einer hohen Basensättigung von weit über $90 \%$ bleibt sie in allen Horizonten der holozänen Parabraunerde relativ gering. Immerhin werden in "Braunerden hoher Basensättigung aus Würmlöß" und in fossilen Lößböden S-Werte bis zu $27 \mathrm{mval} / 100 \mathrm{~g}$ erreicht (vgl. Heide 1954: 84-100). Doch zeigt die Heidesche Bodentypenkarte des Abbaugebietes Fortuna-Nord auch, daß trotz gleichbleibender Bodenart (feinsandiger Lehm) erhebliche Standortunterschiede bestehen, die sich typologisch und bodenchemisch auswirken, und die die Rekultivierungseignung begrenzen können.

Was die Gehalte an organischer Substanz im Profil Garzweiler betrifft, so zeigt sich die zu erwartende Abnahme mit zunehmender Entfernung von der heutigen Oberfläche. In den Fossilböden dürfte der nicht mineralisierte Humus fest an die Tonfraktion gebunden sein.

\section{Beurteilung der Rekultivierungseignung}

Im Rheinischen Braunkohlentagebau werden große Flächen der landwirtschaftlichen Nutzung entzogen und nach Auskohlung eines Tagebaus rekultiviert (s. Tab. 1). Für eine ordnungsgemäße Rekultivierung ist sowohl eine ausgereifte Technik als auch die genaue Beurteilung der zur Verfügung stehenden Bodenmaterialien notwendig. Ersteres besteht in einer getrennten Ab- und Auftragung verschiedenartiger Rekultivierungsmaterialien sowie in einer schonenden Umlagerung. Die Beurteilung der Boden- bzw. Sedimenteigenschaften muß sich zunächst am Verwendungszweck der Neulandflächen orientieren. Für eine landwirtschaftliche Nutzung müssen daher die Anforderungen von Kulturpflanzen herangezogen werden. Neben quantitativen kommt auch den qualitativen Parametern besondere Bedeutung zu, die durch die Rekultivierungstechnik in hohem Maße negativ beeinflußt werden können. Da Lösse im allgemeinen gut komprimierbar sind und zudem der Rekultivierungserfolg und damit auch der Wert dieser Kultosole in erster Linie an den bodenphysikalischen Eigenschaften bemessen wird (vgl. SCHRÖDER 1988), sind diese Parameter im Gegensatz zu den bodenchemischen und -biologischen von besonderer Relevanz.

Für das Lößprofil Garzweiler schwankt die rechnerisch ermittelte effektive Lagerungsdichte (AG Bodenkunde 1982: 125-128) zwischen $1,50 \mathrm{~g} / \mathrm{cm}^{3}$ und $1,75 \mathrm{~g} / \mathrm{cm}^{3}$ und ist damit ausschließlich in die Klasse Ld3 (mittel) einzuordnen. Die Werte der ge- 
Tab. 3: Bodenchemische Parameter

\begin{tabular}{|c|c|c|c|c|c|c|c|c|c|}
\hline Horizont & Tiefe & $\begin{array}{c}\mathrm{pH} \\
\left(\mathrm{CaCl}_{2}\right)\end{array}$ & $\mathrm{CaCO}_{3}$ & $\mathrm{C}_{\text {org }}$ & $\mathrm{KAK}_{\mathrm{eff}}$ & $\mathrm{Ca}^{++}$ & $\begin{array}{l}\text { tionen- } \\
\mathrm{Mg}^{++}\end{array}$ & $\mathrm{K}^{+}$ & $\mathrm{Na}^{+}$ \\
\hline & $\mathrm{cm}$ & & $\%$ & $\%$ & \multicolumn{5}{|c|}{$\mathrm{mval} / 100 \mathrm{~g}$} \\
\hline Ap & $0-33$ & 6,32 & & 0,9 & 10,1 & 8,2 & 0,4 & 0,6 & 0,1 \\
\hline $\mathrm{Bt}$ & -60 & 6,63 & - & 0,3 & 11,7 & 10,0 & 0,6 & 0,4 & 0,1 \\
\hline Bvt & -115 & 6,80 & - & 0,1 & 11,0 & 9,3 & 0,7 & 0,2 & 0,1 \\
\hline $\mathrm{Bbt}$ & -178 & 6,92 & - & 0,1 & 9,7 & 8,2 & 0,8 & 0,2 & 0,1 \\
\hline Bv & -190 & 7,02 & - & 0,1 & 10,8 & 9,0 & 1,1 & 0,2 & 0,1 \\
\hline $\mathrm{C}$ & -280 & 7,80 & 14,5 & 0,1 & 10,4 & 10,8 & 0,8 & 0,2 & 0,1 \\
\hline 1.fGcoCv & -375 & 7,84 & 16,9 & 0,1 & 10,7 & 11,9 & 0,9 & 0,2 & 0,1 \\
\hline fGcoC & -400 & 7,85 & 19,2 & 0,1 & 9,1 & 10,4 & 0,9 & 0,1 & 0,1 \\
\hline C & -640 & 7,85 & 16,7 & 0,1 & 8,8 & 10,2 & 0,9 & 0,2 & 0,1 \\
\hline 2. $\mathrm{fGoC}$ & -700 & 7,87 & 13,6 & 0,1 & 11,8 & 11,9 & 1,4 & 0,2 & 0,2 \\
\hline $\mathrm{fGoCv}$ & -745 & 7,80 & 10,4 & 0,1 & 13,0 & 12,5 & 1,7 & 0,2 & 0,2 \\
\hline fGroCv & -780 & 7,80 & 4,9 & 0,1 & 13,6 & 12,8 & 2,0 & 0,2 & 0,2 \\
\hline 3. $f B v$ & -860 & 7,57 & - & - & 11,4 & 9,3 & 2,0 & 0,2 & 0,1 \\
\hline
\end{tabular}

sättigten Wasserleitfähigkeit sind nach AG BODENKUNDE (1982: 153) für den carbonathaltigen Weichsellöß als mittel, für den carbonatfreien Löß (mit Ausnahme des Bvt-Horizontes) als hoch bis sehr hoch zu klassifizieren. Als weiteres Merkmal zur Charakterisierung des Bodengefüges ist die nutzbare Feldkapazität anzuführen (Porendurchmesser 50 $0,2 \mu \mathrm{m})$. Dieser Porenanteil variiert zwischen $22,7 \%$ (Bt-Horizont) und 40,9\% (liegender C-Löß des 1. Tundragleys). Für eine effektive Durchwurzelungstiefe von $10 \mathrm{dm}$ resultieren daraus nutzbare Feldkapazitäten von 227 bis $409 \mathrm{~mm}$, welche nach der fünfstufigen Skala (AB BODENKUNDE 1982: 150) als sehr hoch eingeordnet werden.

Obwohl bodenphysikalische und auch bodenchemische Unterschiede im Lößprofil Garzweiler festzustellen sind, diese aber nur z. T. in der Klassifizierung zum Ausdruck kommen (s. o.), erweisen sich alle untersuchten Horizonte als gut bis sehr gut geeignetes Rekultivierungsmaterial. Da der im Rheinischen Braunkohlentagebau anstehende Weichsellöß in einer Mächtigkeit von jeweils 4 bis $6 \mathrm{~m}$ abgetragen wird, entsteht ein Gemisch, dessen Primäreigenschaften von den ungestörten holozänen Böden und carbonathaltigen Lössen sowie sekundär durch die Mischung und Umlagerung bestimmt werden. Unterstellt man aus qualitativer und quantitativer (!) Sicht eine homogene Mischung, so ist dieses Löß-Boden-Gemisch ebenso wie die einzelnen Horizonte des Profils Garzweiler für einen Kulturpflanzenstandort als gut geeignet zu beurteilen.

Durch den Vergleich der vorliegenden Ergebnisse mit Kultosolen unterschiedlicher Bewertung (SCHRÖDER 1988: Tab. 2 u. 3; SCHRÖDER \& STEPHAN \& SCHUL-
TE-KARring 1985: Tab. 2) ist festzustellen. daß die Bodenstruktur durch das Umlagerungsverfahren intensiv gestört werden kann. Die mechanische Belastung und vor allem die durch hohe Bodenfeuchtigkeit verminderte Stabilität der Löß-Boden-Gemische führt zu einer Reduzierung des Gesamtporenvolumens bis auf 35,1\% (SCHRÖDER 1988: Tab. 2). Mit der vorliegenden differenzierten Untersuchung des ungestörten Weichsellösses wird erkennbar, daß ungünstige Bodeneigenschaften von Kultosolen nicht ausschließlich auf eine unsachgemäße Rekultivierungstechnik oder fehlerhafte Bodenbearbeitung zurückzuführen sind, sondern durchaus mit den Primäreigenschaften der Lösse erklärt werden können. So weisen Schröder \& Stephan \& Schulte-KarRING (1985: Tab. 2: Profil 2) ein schlecht rekultiviertes Profil mit einem Grobporenvolumen von 6,2\% aus, das genau dem Wert des fGoCv-Horizontes im Profil Garzweiler entspricht.

Tendenziell ist ersichtlich, daß die Lagerungsdichten der Kultosole stets höher ( 1,47 bis $\left.1,85 \mathrm{~g} / \mathrm{cm}^{3}\right)$ sowie die gesättigten Wasserleitfähigkeiten vielfach geringer sind $(0,8$ bis $38,9 \mathrm{~cm} / \mathrm{d}$ im Unterboden $)$ als beim ungestörten Weichsellöß (SCHRÖDER \& STEPHAN \& Schulte-Karring 1985: Tab. 2). Dies unterstützt die Annahme, daß rekultivierte Böden z. T. erhebliche Porendiskontinuitäten aufweisen. Dennoch können mit den guten Primäreigenschaften der zur Verfügung stehenden Lösse und der mittlerweile ausgereiften Rekultivierungstechnik (SCHRÖDER 1988: 2) im Rheinischen Braunkohlentagebau Neulandböden erstellt werden, die durch eine die Rekultivierung abschließende Bodenschätzung mit Bodenzahlen zwischen 50 und 70 Punkten bewertet werden. 


\section{Ausblick}

Löß bedeckt als periglaziales Staubsediment etwa $20 \%$ der heutigen Landoberfläche (CATT 1992: 52). Aus ihm haben sich die ertragreichsten Böden der Erde entwickelt.

Im Rheinischen Braunkohlentagebau werden daher kalkhaltige Weichsellösse im Gemisch mit den holozänen Böden zur Herstellung von Kultosolen verwendet. Da die hier anstehenden holozänen, nicht hydromorphen Böden und der weichselzeitliche Löß fast ausnahmslos günstige bis sehr günstige Eigenschaften aufweisen, ist der Umlagerung und Auftragung, aber auch der Bodenbearbeitung besondere Bedeutung beizumessen.

\section{Schriftenverzeichnis}

AG Bodenkunde (1982): Bodenkundliche Kartieranleitung. - 331 S., 19 Abb., 98 Tab.; Hannover (E. Schweizerbart'sche Verlagsbuchhandlung).

BRONGER, A. (1974): Zur postpedogenen Veränderung bodenchemischer Kenndaten insbesondere von pedogenen Eisenoxiden in fossilen Lößböden. - Transact. 10th Internat. Congr. Soil Sci., V: 429-441, 1 Abb., 2 Tab; Moskau.

BrunNaCKer, K. \& HAHN, J. (1978): Der jungpleistozäne Löß samt paläolithischen Kulturen in den Rheinlanden als Glied einer zeitlichen und räumlichen Faziesänderung. - In: NAGL, H. (Hrsg.): Beiträge zur Quartär- und Landschaftsforschung. Festschrift zum 60. Geburtstag von Julius Fink, XVI + 687 S.; Wien (Hirt).

CATT, J. A. (1992): Angewandte Quartärgeologie. - 358 S., 129 Abb., 14 Tafeln, 31 Tab.; Stuttgart (Enke).

Eggert, P., Hübener, J. A., Prifm, J., Stein, V., Vossen, K. \& WetTig, E. (1986): Steine und Erden in der Bundesrepublik Deutschland - Lagerstätten, Produktion und Verbrauch. - Geol. Jb., D 82: 879 S., 17 Abb., 156 Tab.; Hannover (Schweizerbart'sche Verlagsbuchhandlung).

Hase, D. \& Meyer, B. (1969): Feuchte-Jahresgang, WasserBewegungen und -Bilanzen in dicken WürmlößDecken und ihren holozänen Böden (Parabraunerde, Griserde, Feuchtschwarzerde) bei unterschiedlichem Grundwasserstand im Raum Niedersachsen. - Göttinger Bodenkundl. Ber., 11: 85-183, 7 Abb., 7 Tab.; Göttingen.

HEIDE, G. (1954): Die bodenkundlichen Voraussetzungen für die landwirtschaftliche Rekultivierung der Braunkohlentagebaue in der Ville. - Diss. Univ. Bonn, $105 \mathrm{~S}$, $13 \mathrm{Abh}$.

HOCHT, F. VON DER (1990): Im Rheinischen Braunkohlenrevier anstehendes, für die Rekultivierung nutzbares Bo- denmaterial. - Braunkohle, 42: 11-15, 4 Abb., 3 Tab.; Düsseldorf (Zeitschriftenverlag RBDV).

Klostermann, J. (1992): Das Quartär der Niederrheinischen Bucht. - 200 S., 30 Abb., 8 Tab., 2 Tafeln; Krefeld (Geol. Landesamt).

LANDESOBERBERGAMT NW (1973): Richtlinien des Landesoberbergamts Nordrhein-Westfalen für das Aufbringen von kulturfähigem Bodenmaterial bei landwirtschaftlicher Rekultivierung für die im Tagebau betriebenen Braunkohlenwerke vom 21. 1. 1973 in der Fassung vom 2. 3. 1984. - 4 S.; Dortmund.

LAUTRIDOU, J. P., MASSON, M. \& VOIMENT, R. (1987): Loess et geotechnique: l'exemple des limons de Normandie. Catena Supplement, 9: 11-25, 9 Fig.; Cremlingen-Destedt (Catena).

LuFT, G. (1980): Abfluß und Retention im Löß, dargestellt am Beispiel des hydrologischen Versuchsgebietes Rippach, Ostkaiserstuhl. - Beiträge zur Hydrologie, Sonderheft 1, 241 S., 51 Abb., 25 Tab., 65 Anlagen; Kirchzarten (Nippes).

PAAS, W. (1968): Stratigraphische Gliederung des Niederrheinischen Lösses und seiner fossilen Böden. - Decheniana, 121: 9-18, 10 Abb., 2 Tab.; Bonn (Selbstverlag des Naturhistorischen Vereins).

RICKEN, W. (1983): Mittel- und jungpleistozäne Lößdecken im südwestlichen Harzvorland. Stratigraphie, Paläopedologie, fazielle Differenzierung und Konnektierung in Flußterrassen. - Catena Supplement, 3: 95-138, 12 Abb., 2 Tab.; Cremlingen-Destedt (Catena).

Rohdenburg, H. \& Meyer, B. (1966): Zur Feinstratigraphie und Paläopedologie des Jungpleistozäns nach Untersuchungen an südniedersächsischen und nordhessischen Lößprofilen. - Mitteilgn. Dtsch. Bodenkundl Gesellschaft, 5: 1-135, 25 Abb., 12 Tab.; Göttingen.

SCHRÖDER, D. (1988): Bodenschonende Rekultivierung von Lößböden in Braunkohletagebauen. - In: ROSENKRANz, D., Einsele, G. \& Harress, H.-M. (Hrsg.): Bodenschutz. Ergänzbares Handbuch der Maßnahmen und Empfehlungen für Schutz, Pflege und Sanierung von Böden, Landschaft und Grundwasser, 2. Band, 7230, 1. Lfg. XI/88, 22 S., 3 Abb., 5 Tab.; Berlin (Erich Schmidt Verlag).

SCHröder, D., StephaN, S. \& SCHUlte-KARring, H. (1985): Eigenschaften, Entwicklung und Wert rekultivierter Böden aus Löß im Gebiet des Rheinischen Braunkohlen-Tagebaues. - Z. Pflanzenernaehr. Bodenkd., 148 131-146, 1 Abb., 5 Tab.; Weinheim (VCH Verlagsgesellschaft).

STARKE, R. (1990): Wiedernutzbarmachung im Rheinischen Braunkohlenrevier. - Braunkohle, 42: 4-10, 4 Abb., 1 Tab.; Düsseldorf (Zeitschriftenverlag RBDV).

Tillmanvs, W. \& Brunnacker, K. (1987): The Lithology and Origin of Loess in Western Central Europe. - Catena Supplement, 9: 47-54, 7 Fig., 1 Tab.; Cremlingen-Destedt (Catena).

Manuskript eingegangen am 18. 1. 1993 